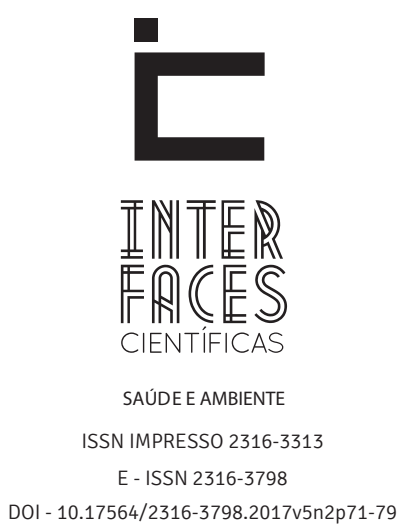

\title{
INFECÇ̃̃̃ DO SITITO CIRÚRGICO EM PACIENTES NO PÓS-OPERATÓRIO DE CIRURGIAS ORTOPÉDICAS ELETIVAS
}

\author{
SURGICAL SITE INFECTION IN PATIENTS ON POSTOPERATIVE ELECTIVE ORTHOPEDIC SURGERY \\ INFECCIÓN DEL SITIO QUIRÚRGICO EN PACIENTES EN POSTOPERATORIO DE CIRUGíAS ORTOPÉDICAS ELECTIVAS
}

Paulo Vitor Ferreira Santos ${ }^{1}$

Kamilla Ismerim Santos Pereira de Santana ${ }^{3}$

Luciana Santos Cariri ${ }^{5}$
Karoline Bispo de Jesus ${ }^{2}$ Emília Cervino Nogueira ${ }^{4}$

Fabiana Pereira Guimarães Brito ${ }^{6}$

\section{RESUMO}

A Infecção do Sítio Cirúrgico (ISC) é definida como um processo infeccioso inflamatório da ferida ou cavidade operatória. 0 estudo teve como objetivo identificar a incidência de infecção do sitio cirúrgico no período pós-operatório em pacientes submetidos a cirurgias eletivas ortopédicas, classificar o tipo de cirurgia quanto ao potencial de contaminação e caracterizar a ferida operatória quanto ao grau de infecção. Estudo descritivo de caráter exploratório com abordagem quantitativa, realizado com 140 pacientes durante consulta em pós-operatório de cirurgia ortopédica no ambulatório de retorno localizado em um Hospital Geral do Estado de Sergipe. Dos 140 pacientes que compareceram ao retorno no pós-operatório de cirurgia ortopédica, 122 (80\%) foram do sexo masculino e $28(20 \%)$ do sexo feminino. Quanto a faixa etária, 131 (93,6\%) pacientes encontravam-se entre 18-59 anos, e 9 (6,4\%) acima dos 60 anos. A maior parte das cirurgias ortopédicas foi classificada como cirurgias limpas, 129 (92,10\%), e apenas 11 (7,90\%) foram classificadas como infectadas. Foram detectadas 78 ISC (55,8\%), sendo que 60 dessas infecções $(42,9 \%)$ foram classificadas como infecção do sítio cirúrgico incisional superficial e 18 infecções (12,9\%) classificadas como infecção do sítio cirúrgico incisional profunda. Na relação entre a classificação cirúrgica e a classificação da infecção do sítio cirúrgico, o estudo mostrou que entre as cirurgias limpas, 62 procedimentos $(48,1 \%)$ não apresentaram sinais de infecção. A diabetes mellitus, a hipertensão, a cirurgia prolon- 
gada, o uso de fixador externo, o tempo pré-operatório prolongado e o uso de material de implante foram identificados como fatores de risco para ISC.

\section{ABSTRACT}

The Surgical Site Infection (SSI) is defined as an inflammatory infectious process of the wound or surgical cavity. The study aimed to identify the incidence of surgical site infection in the postoperative period in patients undergoing elective orthopedic surgery, classify the type of surgery and the potential for contamination and characterize the wound on the degree of infection. Descriptive exploratory study with a quantitative approach, carried out with 140 patients during consultation on post-operative orthopedic surgery in return clinic located in a general hospital of the state of Sergipe. Of the 140 patients who attended the return postoperative orthopedic surgery, $122(80 \%)$ were male and 28 (20\%) were female. As for age group, 131 (93.6\%) patients were between 18-59 years, and 9 (6.4\%) over 60 years. Most orthopedic surgeries were classified as clean 129 (92.10\%), and only 11

\section{PALAVRAS-CHAVE}

Infecção da Ferida Operatória. Período Perioperatório. Ortopedia.

(7.90\%) were classified as infected. $78 \mathrm{SSI}$ were detected (55.8\%), 60 of these infections (42.9\%) were classified as surgical site infection superficial incisional and 18 (12.9\%) classified as surgical site infection deep incisional. The relationship between surgical classification and the classification of surgical site infection, the study showed that among the clean surgeries, 62 procedures (48.1\%) showed no signs of infection. Diabetes Mellitus, hypertension, prolonged surgery, the use of external fixation, prolonged preoperative time and use of the implant material were identified as risk factors for SSI.

\section{KEYWORDS}

Surgical Wound Infection. Perioperative period. Orthopedics.

\section{RESUMEN}

La Infección del Sitio Quirúrgico (ISQ) se define como un proceso infeccioso inflamatorio de la herida o de la cavidad quirúrgica. El estudio tuvo como objetivo identificar la incidencia de infección del sitio quirúrgico en el postoperatorio en pacientes sometidos a las cirugías electivas ortopédicas, clasificar el tipo de cirugía cuanto al potencial de contaminación y caracterizar la herida en el grado de infección. Estudio exploratorio descriptivo con abordaje cuantitativo, realizado con 140 pacientes durante la consulta en la cirugía ortopédica postoperatorio en la clínica de retorno situado en un Hospital General del estado de Sergipe. De los 140 pacientes que asistieron al retorno en el postoperatorio de cirugía ortopédica, 122 (80\%) eran hombres y 28 (20\%) eran mujeres. En cuanto a grupos de edad, 131 (93,6\%) pacientes tenían entre 18-59 años, y 9 (6,4\%) mayores de 60 años. La mayoría de las cirugías ortopédicas fueron clasificadas como limpia 129 (92,10\%), y solo $11(7,90 \%)$ fueron clasificados como infectados. Fueron detectados 78 ISC (55,8\%), 60 de estas infecciones $(42,9 \%)$ fueron clasificadas como infección del sitio quirúrgico superficial incisional y 18 infecciones (12,9\%) clasificados como infección del sitio quirúrgico incisional profundo. En la relación entre la clasificación quirúrgica y la clasificación de la infección del sitio quirúrgico, el estudio mostró que entre las cirugías limpias, 62 procedimientos $(48,1 \%)$ no mostraron signos de infección. La diabetes 
mellitus, la hipertensión, la cirugía prolongada, el uso de la fijación externa, el tiempo preoperatorio prolongado y uso de material de implante fueron identificados como factores de riesgo de ISC.

\section{INTRODUÇ̃̃O}

A infecção hospitalar é um termo que define toda infecção adquirida na admissão, na permanência e na alta do paciente em um serviço de saúde hospitalar, considerada mundialmente um problema de saúde pública e classificada conforme sua tipologia em infecção respiratória, vascular, urinária e infecção do sítio cirúrgico (ISC). Neste sentido, a ISC é considerada uma infecção hospitalar por se tratar de infecção adquirida em procedimento cirúrgico com um tempo de encubação que varia de 3 a 8 dias, e corresponde a $20 \%$ dos casos segundo a National Nosocomial Infection Surveillance System (NNIS) (AGUIAR ET AL., 2012).

A ISC é definida como um processo infeccioso inflamatório da ferida ou cavidade operatória e destaca-se como um dos principais tipos de infecção na assistência em pacientes cirúrgicos hospitalizados. Ocupando a terceira posição com 14 a 16\% dos casos entre as Infecções Relacionadas à Assistência à Saúde (IRAS), a ISC pode ser diagnosticada até 30 dias após a cirurgia ou até um ano em procedimentos que envolvem o implante de prótese (BATISTA, RODRIGUES, 2012). Nos Estados Unidos são realizados em média 16 milhões de procedimentos cirúrgicos anualmente, apresentando um percentual de 2 a $5 \%$ de ISC, enquanto que no Brasil a situação difere da realidade norte-americana, apresentando incidência de 14 a $16 \%$ em pacientes hospitalizados (ERCOLE ET AL., 2011; GEBRIM ET AL., 2014).

Uma série de fatores pode ser considerada na etiologia da ISC, como o preparo ineficiente do paciente no pré-operatório, condição clínica do paciente, tempo prolongado no período pré-operatório, demora do procedimento cirúrgico, falha no processo de antissepsia, degermação ineficaz do médico cirurgião e

\section{PALABRAS CLAVE}

La infección de herida quirúrgica, Preoperatorio; Ortopedia.

da equipe, ambiente da sala cirúrgica e materiais de implantes em procedimentos cirúrgicos ortopédicos (ERCOLE, FRANCO, 2011; LONGO, RIBEIRO, 2011).

As infecções ligadas aos procedimentos ortopédicos destacam-se entre as ISC, e são consideradas de altíssima gravidade, pois envolvem o uso de materiais de implantes, potencializando o risco do processo infeccioso que pode acarretar em sérias complicações ao paciente desde a perda do membro operado até a morte. Outras consequências evidenciadas são o aumento do tempo de internação nosocomial, aumento da taxa de reospitalização e o aumento do custo hospitalar em 300\% (ERCOLE ET AL., 2011; RIBEIRO ET AL., 2013).

A infecção do sítio cirúrgico classifica-se em três tipos: incisional superficial, que envolve somente 0 tecido subcutâneo e pode apresentar secreção purulenta na incisão; incisional profunda, que atinge tecidos moles, músculos, fáscia e apresenta secreção de aspecto purulento, deiscência espontânea e médica, e órgão ou cavidade na qual ossos e órgãos são atingidos (BATISTA, RODRIGUES, 2012).

A classificação da tipologia cirúrgica quanto ao grau de contaminação está ligada ao tecido operado, a presença ou ausência de flora bacteriana, dentre outros fatores. Dessa forma, as cirurgias podem ser classificadas em cirurgia limpa, cirurgia potencialmente contaminada, cirurgia contaminada e cirurgia infectada (AGUIAR ET AL., 2012).

O momento cirúrgico é expresso por terminologias específicas de acordo com o seu tempo. 0 agendamento do procedimento cirúrgico, a cirurgia propriamente dita e a saída do paciente da sala cirúrgica compreendem o período perioperatório, e de acordo com o momento esse período pode ser classificado em período 
pré-operatório, intraoperatório e pós-operatório, períodos esses que têm grande valia no aparecimento das infecções do sítio cirúrgico (DUAILIBE ET AL., 2014).

A assistência de enfermagem é essencial após o procedimento cirúrgico, onde se inicia o período pós-operatório. Suas intervenções se baseiam na prevenção e no tratamento de agravos oriundos de procedimentos cirúrgicos. Cabe ao enfermeiro fazer uma avaliação sistemática desses pacientes e prestar cuidados no pós-operatório (ROCHA, MORAES, 2010).

A cirurgia eletiva, definida como aquela que se pode agendar o dia escolhido para sua realização, livre de caráter urgente ou emergente que necessitam de certo imediatismo, estão rodeadas de critérios médicos para sua realização que vão desde a solicitação de exames laboratoriais até pareceres de outros profissionais, evitando assim o risco de possíveis intercorrências (CARVALHO ET AL., 2010).

É imprescindível, portanto, a atuação do enfermeiro na prestação de cuidados preventivos à infecção, sendo esta uma das complicações que mais acomete o paciente cirúrgico devido a distúrbios fisiológicos, patológicos, cuidados ou educação deficiente tanto da equipe de enfermagem quanto da família.

Diante do exposto, o presente estudo teve como objetivo identificar a incidência de infecção do sitio cirúrgico no período pós-operatório em pacientes submetidos a cirurgias eletivas ortopédicas, classificar o tipo de cirurgia quanto ao potencial de contaminação além de caracterizar a ferida operatória quanto ao grau de infecção.

\section{METODOLOGIA}

Tratou-se de um estudo descritivo de caráter exploratório com abordagem quantitativa, realizado no ambulatório de retorno da ortopedia, localizado em um Hospital Geral do Estado de Sergipe.

A pesquisa foi realizada com todos os pacientes atendidos na consulta médica em pós-operatório de cirurgia ortopédica durante o período de coleta (140 pacientes). Os critérios para a inclusão foram: pacientes submetidos a cirurgias eletivas ortopédicas; idade maior que 18 anos e consentir livremente a sua participação no estudo. Os critérios para exclusão foram: pacientes submetidos a cirurgias ortopédicas de urgência e emergência.

A coleta de dados foi realizada no mês de novembro do ano de 2015, durante os turnos matutino e vespertino, de acordo com os critérios de inclusão. Os dados foram coletados pelos pesquisadores, utilizando um roteiro para coleta de dados, registrando as seguintes informações: dados de identificação do paciente; dados relacionados à cirurgia; dados referentes a fatores de risco; critérios de infecção segundo a literatura e classificação da infecção do sítio cirúrgico.

As variáveis do estudo foram analisadas por meio do programa estatístico StatisticalPackage for the Social Sciences (SPSS) 21.0, por estatística descritiva, utilizando análise de frequência absoluta e relativa. Todas as análises estatísticas foram realizadas com o intervalo de confiança igual ou menor que $5 \%$.

Os dados foram coletados após a autorização do Comitê de Ética em Pesquisa em Seres Humanos da Universidade Tiradentes com o parecer de $n^{\circ}$ 1.304.422. O presente estudo resguardou todos os direitos e identidade dos participantes desta pesquisa atendendo a Resolução $n^{0} 466$ de dezembro de 2012, do Conselho Nacional de Saúde do Ministério de Saúde - Brasília/DF.

\section{RESULTADOS E DISCUSSÃO}

Dos 140 pacientes que compareceram ao retorno no pós-operatório de cirurgia ortopédica, 122 (80\%) foram do sexo masculino e $28(20 \%)$ do sexo feminino. Quanto a faixa etária, 131 (93,6\%) pacientes encontravam-se entre 18-59 anos, e 9 $(6,4 \%)$ acima dos 60 anos.

Características do paciente como idade e sexo podem ajudar a explicar a incidência de infecção do sítio cirúrgico (ISC). É observado nos últimos 
tempos um número maior de internação de pacientes idosos por intercorrências em procedimento cirúrgico. Fato preocupante, porque o internamento dos idosos após o procedimento cirúrgico potencializa o aparecimento da ISC, em função das várias alterações da senilidade, da queda brusca, da resposta imunológica e dos diversos procedimentos invasivos realizados (LENARDT ET AL., 2010).

Para Ampuero e Dios (2010), o fator idade é um dado a ser investigado, mas segundo Triantafyllopoudos e outros autores (2015), o risco de desenvolver uma infecção do sítio cirúrgico em procedimentos ortopédicos é maior em pacientes idosos em comparação com pacientes jovens que passaram pelo mesmo tipo de procedimento. Os idosos apresentam características, como a resposta imunitária deficiente, agentes infecciosos, estado de desnutrição e as demais comorbidades já existentes no idoso, contribuindo desta forma para o aparecimento da ISC.

Pacientes com idade inferior a 44 anos foram identificados como fator de risco para ISC em procedimentos ortopédicos, comparados com pacientes com idade entre 45-65 anos. Uma explicação plausível para esse fato seria um aumento de cirurgias em jovens para tratamento de doenças inflamatórias com implante de próteses articulares, osteoartrite pós-traumática após fratura exposta, imunossupressão e hemofilia (VALLES ET AL., 2012).

Os dados relacionados à cirurgia, aos fatores de risco e aos critérios e classificação da infecção do sítio cirúrgico foram organizados e analisados em categorias, conforme descrito a seguir:

\subsection{CLASSIFICAÇ̃̃O CIRÚRGICA QUANTO AO GRAU DE CONTAMINAÇÃO}

A maior parte das cirurgias ortopédicas foi classificada como cirurgias limpas 129 (92,10\%), e apenas 11 (7,90\%) foram classificadas como infectadas. Não houve relatos sobre cirurgias potencialmente contaminada (FIGURA 1).
Figura 1 - Percentual de classificação de cirurgia quanto ao grau de contaminação $(n=140)$

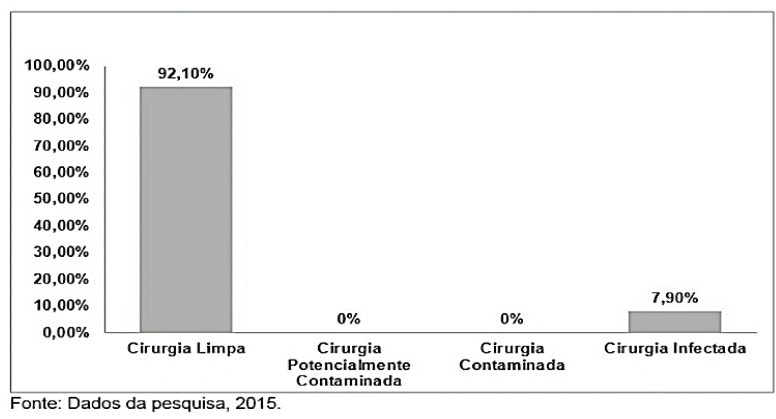

Fonte: Dados da pesquisa;

O risco de infecção aumenta proporcionalmente com o grau de contaminação da ferida operatória. Segundo a probabilidade de contaminação as cirurgias podem ser classificadas em cirurgias limpas, que são estéreis, com fechamento por primeira intenção, isenta de infecção e não há falha na técnica asséptica; cirurgias potencialmente contaminadas, na qual ocorre a abertura do trato respiratório, gastrointestinal ou geniturinário, sem sinais de inflamação; cirurgias contaminadas, que apresentam infecção não purulenta, falha na técnica asséptica, trauma exposto a menos de 4 horas e infecção do trato gastrointestinal; infectadas, com presença de secreção purulenta, tecido desvitalizado ou corpo estranho (ERCOLE ET AL., 2011; AGUIAR ET AL., 2012).

\subsection{CLASSIFICAÇ̃̃O DA INFECÇ̃̃O DO SÍTIO CIRÚRGICO}

Nos 140 pacientes que se submeteram a procedimentos cirúrgicos ortopédicos foram detectadas 78 ISC $(55,8 \%)$, sendo que 60 dessas infecções $(42,9 \%)$ foram classificadas como Infecção do Sítio Cirúrgico Incisional Superficial (ISC - IS) e 18 infecções (12,9\%) classificadas como Infecção do Sítio Cirúrgico Incisional Profunda (ISC - IP). 
No contexto das infecções hospitalares, a infecção do sítio cirúrgico tem sido apontada como um dos mais importantes sítios de infecção que ocorre na incisão cirúrgica ou em tecidos manipulados durante a operação, classificada de acordo com sua topografia em incisional superficial, profunda, e de órgão/espaço (RIBEIRO ET AL., 2013).

Em cirurgias eletivas os cuidados profiláticos de infecção do sitio cirúrgico são mais eficazes comparados com cirurgias de urgência e emergência. É durante o período intraoperatório que ocorre a maioria das ISC, por esse motivo se faz necessário medidas imprescindíveis para o controle da infecção, como antissepsia rigorosa das mãos, paramentação completa, limpeza e esterilização de todos os materiais e evitar campos e aventais molhados, pois devem ser considerados contaminados (LONGO; RIBEIRO, 2011). A profilaxia por meio do uso de antibióticos é recomendada em cirurgias eletivas, ocasionando uma diminuição da bacteremia e, consequentemente, do número de microrganismos próximos ao local da incisão (CARVALHO, 2014).

Na relação entre a classificação cirúrgica e a classificação da infecção do sítio cirúrgico, o estudo mostrou que entre as cirurgias limpas, 62 procedimentos (48,1\%) não apresentaram sinais de infecção (TABELA 1).

Tabela 1 - Percentual da classificação cirúrgica e classificação da infecção do sítio cirúrgico (ISC) $(n=140)$. IS - incisional superficial; IP - incisional profunda

\begin{tabular}{lllll}
\hline \multirow{5}{*}{$\begin{array}{l}\text { Classificação } \\
\text { da Cirurgia }\end{array}$} & ISCIS & ISC IP & $\begin{array}{l}\text { Ausência de } \\
\text { Infecção }\end{array}$ & Total \\
& $n(\%)$ & $n(\%)$ & $n(\%)$ & $n(\%)$ \\
\hline $\begin{array}{l}\text { Cirurgia } \\
\text { Limpa }\end{array}$ & $56(43,4)$ & $11(8,5)$ & $62(48,1)$ & $129(100)$ \\
$\begin{array}{l}\text { Cirurgia } \\
\text { Infectada }\end{array}$ & $4(36,4)$ & $7(63,6)$ & $0(0)$ & $11(100)$ \\
\hline $\begin{array}{l}\text { Fonte: Dados da pesquisa. } \\
\end{array}$
\end{tabular}

Interfaces Científicas - Saúde e Ambiente - Aracaju • V.5 - N.2 • p. 71 - 79 • Fev. 2017

\subsection{FATORES DE RISCO PARA ISC}

O estudo demonstrou que 73 (55,7\%) dos pacientes com faixa etária entre 18-59 anos apresentaram a infecção do sítio cirúrgico (TABELA 2).

Segundo Valles e outros autores (2012), a alta taxa de ISC em procedimentos ortopédicos, em pacientes com menos de 60 anos, está ligado a um aumento de cirurgias para tratamento de doenças inflamatórias com implante de próteses articulares, osteoartrite e cirurgia pós-traumática. Para Wu e outros autores (2014), os pacientes com idades entre 65-75 anos têm 3 vezes mais chances de desenvolver ISC em cirurgias com próteses articulares em comparação com pacientes com idade entre 45-65 anos. Em geral, as idades avançadas dos pacientes podem se associar com a deficiência do estado nutricional e imunidade enfraquecida, resultando em um risco elevado de infecção.

Tabela 2 - Percentual dos fatores de risco para infecção do sítio cirúrgico (ISC)

\begin{tabular}{lll}
\hline Fatores de Risco & ISC & $\begin{array}{l}\text { Ausência } \\
\text { de Infecção } \\
n(\%)\end{array}$ \\
\hline $18-59$ anos & $n(\%)$ & $58(44,3)$ \\
$60-99$ anos & $5(55,5)$ & $4(44,5)$ \\
DiabetesMellitus & $6(60,0)$ & $4(40,0)$ \\
Hipertensão arterial & $9(81,8)$ & $2(18,2)$ \\
Fixador externo & $29(64,5)$ & $16(35,5)$ \\
Material de implantes & $9(40,9)$ & $13(59,1)$ \\
Cirurgia prolongada & $17(58,7)$ & $12(41,3)$ \\
Pré-operatório prolongado & $7(36,8)$ & $12(63,2)$ \\
\hline Fonte: Dados da pesquisa. & &
\end{tabular}

No que se refere a pacientes hipertensos $81,8 \%$ (9/11) apresentaram infecção do sítio cirúrgico. Já a taxa de infecção entre os diabéticos foi de $60 \%$ dos 
casos (6/10) (TABELA 2). Segundo Pugely e colaboradores (2015), a hipertensão pode causar doenças cardiovasculares como a insuficiência cardíaca congestiva, que geralmente dificultam o processo de cicatrização, favorecendo assim o aparecimento da infecção. Triantafyllopoudos e colaboradores (2015) relatam que a infecção no diabetes mellitus está relacionada a hiperglicemia, por causar uma quebra na resposta fisiológica a cargas bacterianas. Mesmo em pacientes sem diagnóstico de diabetes mellitus, mas com glicose $>140 \mathrm{mg} / \mathrm{dL}$, o risco aumenta em 3 vezes para ISC, em cirurgias ortopédicas.

Outro fator de risco estudado foi o tempo prolongado da cirurgia. Nesse sentido, a pesquisa demonstrou que 58,7\% (17/29) das cirurgias apresentaram um tempo cirúrgico maior que 120 minutos. Segundo Pugely e outros autores (2015), o tempo operatório prolongado (maior que 120 minutos) está intimamente ligado ao aparecimento da infecção em pacientes cirúrgico ortopédicos.

Quanto ao uso de material implantável, 59,0\% $(13 / 22)$ não apresentaram infecção do sítio cirúrgico. A presença desse material é caracterizada por sua capacidade de potencializar o aparecimento de uma infecção e diminuir a resistência do sistema imunológico aos agentes microbianos (ERCOLE ET AL., 2011).

Os procedimentos cirúrgicos ortopédicos, atualmente, vêm apresentando uma alta incidência e uma maior complexidade com uso de novas tecnologias, que envolve o desenvolvimento de próteses e equipamentos especiais. No Brasil, a ISC ligada a procedimentos ortopédicos compreende uma taxa que varia de 1,4 a $40 \%$ dos casos. Dentre os fatores predisponentes para o aparecimento da ISC ortopédica destaca-se o implante de próteses. Estudos mundiais revelam que 1 a $5 \%$ destas próteses tornam-se infectadas, podendo trazer comprometimentos irreparáveis ao paciente que pode perder o membro operado ou até mesmo ir à morte (ERCOLE, FRANCO 2011; LEME ET AL., 2011).

As infecções de próteses são caracterizadas e evidenciadas pelos seguintes sinais e sintomas: dor intensa, febre alta, calor, rubor, vermelhidão e secreção na ferida operatória. 0 que vai determinar a apresen- tação clínica da infecção é a virulência do microrganismo envolvido, o tecido atingido e a via de infecção (LIMA; OLIVEIRA, 2010).

Em relação ao período pré-operatório prolongado (acima de 24 horas), 63,1\% (12/19) não desenvolveram infecção. Para Ampuero e Dios (2010) e Triantafyllopoudos e outros autores (2015), o período pré-operatório prolongado é um importante fator de risco para o aparecimento da ISC, pois quanto mais prolongado o tempo de espera para o procedimento cirúrgico, geralmente acima de $24 \mathrm{~h}$, maior será a exposição a bactérias hospitalares, o que não ocorreu no estudo.

\section{CONCLUSÃO}

O estudo demonstrou que a incidência de infecção do sítio cirúrgico em procedimentos ortopédicos foi elevada, apresentando percentual de 55,8\% (78/140), número esse maior que a variável norte-americana que varia de 2 a $5 \%$ e a brasileira de 14 a 16\% (ERCOLE ET AL., 2011). Tal fato comprova a importância da vigilância desse tipo de infecção no serviço de assistência à saúde.

As cirurgias limpas apresentaram alta incidência com 51,9\% (67/129) de ISC, mostrando que mesmo as cirurgias estéreis podem apresentar um número alto de infecção. Quanto à classificação da ISC destacouse a ISC - IS entre as infecções com 42,9\% (60/78) dos casos, infecção essa detectada com alta incidência nos primeiros dias no pós-operatório cirúrgico.

O sexo masculino e a faixa etária entre 18 - 59 anos apresentaram maior incidência de procedimentos cirúrgicos e de aparecimento da ISC comparado com pacientes do sexo feminino e com faixa etária acima de 59 anos, fato esse explicado pelo maior número de pacientes jovens e do sexo masculino que participaram da pesquisa. Já os pacientes que apresentavam comorbidades como a diabetes mellitus e a hipertensão se destacaram, sendo considerados fatores importantes para o aparecimento de uma infecção. 
No que se refere aos aspectos cirúrgicos, a cirurgia prolongada, o uso de fixador externo, o tempo pré-operatório prolongado e o uso de material de implante apontam como fatores importantes para o desenvolvimento da infecção. A concomitância dos fatores de risco supracitados potencializa o aparecimento da ISC em procedimentos ortopédicos.

A enfermagem e a equipe médica têm papel preponderante no aparecimento da ISC, pois podem agir na prevenção de fatores de risco modificáveis como, por exemplo, a limpeza e desinfecção de equipamentos e do ambiente, higienização das mãos, diminuição do tempo pré-operatório, banho pré-operatório, diminuindo a colonização bacteriana da pele, realização da tricotomia e controle de temperatura.

\section{REFERÊNCIAS}

AGUIAR, A.P.L. et al. Fatores associados à infecção de sítio cirúrgico em um hospital na Amazônia Ocidental Brasileira. Rev. SOBECC. v.17, n.3, São Paulo, jul-set. 2012. p.60-70,

AMPUERO, J.C.; DIOS, M. What are the risk factors for infection in hemiarthroplasties and total hip arthroplasties? Rev. ClinOrthopRelat Res., v.468, n.12, Philadelphia, jun/dez. 2010. p.3268-3277.

BATISTA, T.F.; RODRIGUES, M.C.S. Vigilância de infecção de sítio cirúrgico pós-alta hospitalar em hospital de ensino do Distrito Federal, Brasil: estudo descritivo retrospectivo no período 2005-2010.

Epidemiol. Serv. Saúde, v.21, n.2, Brasília, abr-jun. 2012. p.253-264.

CARVALHO, R.L.R. Fatores de risco para infecção de sítio cirúrgico em procedimentos gerais em um hospital público de Belo Horizonte. 2014.

Dissertação (Mestrado em Enfermagem) - Escola de Enfermagem, Universidade Federal de Minas Gerais, Belo Horizonte, 2014.
CARVALHO, R.W.F. et al. O paciente cirúrgico. Parte I. Rev. Cir. Traumatol. Buco-Maxilo-Fac. v.10, n.4, Camaragibe, out-dez. 2010. p.85-92.

DUAILIBE, F.T. et al. Intervenções de enfermagem na recuperação pós-anestésica de pacientes cirúrgico.

Rev. Enferm. UFPI. v.3, n.1, Teresina, jan./mar. 2014. p.107-112.

ERCOLE, F.F.; FRANCO, L.M.C. Infecção de sítio cirúrgico em pacientes submetidos a cirurgias ortopédicas em um hospital público de Minas Gerais. Rev. Min. Enferm., v.15, n.3, Belo Horizonte, jul-set. 2011. p.399-405.

ERCOLE, F.F. et al. Risco para infecção de sítio cirúrgico em pacientes submetidos a cirurgias ortopédicas. Rev. Latino-Am. de Enfermagem, v.19, n.6, São Paulo, Nov-dez. 2011.

GEBRIM, C.F.L. et al. Análise da profilaxia antimicrobiana para a prevenção da infecção do sítio cirúrgico em um hospital do centro-oeste brasileiro. Ciencia y Enfermeria, n.2, p.103-115, Concepcion, jul-abr. 2014.

LEME, L.E.G. et al. Cirurgia ortopédica em idosos: aspectos clínicos. Rev. Bras. Ortop., v.46, n.3, São Paulo, set-dez. 2011. p.238-246.

LENARDT, M.H. et al. Fatores de risco para mortalidade de idosos com infecção do sítio cirúrgico. Rev. Bras. Geriatr. Gerontol., v.13, n.3, Rio de Janeiro, jun0ago. 2010. p.383-393.

LIMA, M.; OLIVEIRA, P.R.D. Atualização em infecções em próteses articulares. Rev. Bras. Ortop., v.45, n.6, São Paulo, abril 2010. p.520-523.

LONGO, A.R.T.; RIBEIRO, D.R. Hipotermia como fator de risco para infecção de sítio cirúrgico: conhecimento dos profissionais de enfermagem de nível médio. Rev. Min. Enferm., v.15, n.1, Belo Horizonte, jan-mar. 2011. p.34-41. 
PUGELY, A.J. et al. The incidence of and risk factors for 30-day surgical site infections following primary and revision total joint arthroplasty. J Arthroplasty. New York, dez-jan. 2015.

RIBEIRO, J. C. et al. Ocorrência e fatores de risco para infecção de sítio cirúrgico em cirurgias ortopédicas. Acta Paul. Enferm., v.26, n.4, São Paulo, jul-ago. 2013. p.353-359.

ROCHA, L.S.; MORAES, M.W. Assistência de enfermagem no controle da dor na sala de recuperação pós-anestésica. Rev. Dor, v.11, n.3, São Paulo, jul-set. 2010. p.254-258.
TRIANTAFYLLOPOUDOS, G. et al. Patient, surgery, and hospital related risk factors for surgical site infections following total hip arthroplasty. Scientific World Journal, New York, set-jan. 2015. p.1-9.

VALLES, A.G.D. et al. In-hospital surgical site infections after primary hip and knee arthroplasty incidence and risk factors. J Arthroplasty, v.28, New York, jan-jun. 2012. p.385-389.

WU, C. et al. Risk factors for periprosthetic joint infection after total hip arthroplasty and total knee arthroplasty in chinese patients. Plos One, v.9, n.4, San Francisco. abr. 2014. p.1-7.

4 Mestre em Ciências da Saúde pela Universidade Federal de Sergipe UFS; Docente do curso de Enfermagem da Universidade Tiradentes - UNIT, Aracaju-SE. Email: ecervino.n@ig.com.br

5 Especialista em emergência e UTI pela Faculdade Social da Bahia; Docente do curso de Enfermagem da Universidade Tiradentes - UNIT, Aracaju-SE. Email: lucianasantosca@yahoo.com

6 Mestre em Saúde e Ambiente pela Universidade Tiradentes - UNIT; Docente do curso de Enfermagem da Universidade Tiradentes - UNIT, Aracaju-SE. Email: fabibritoenf@yahoo.com.br 


\section{PRODUCÃÃO}

Editora Universitária Tiradentes

\section{IMPRESSÃO}

\section{Gráfica Gutemberg}

Universidade Tiradentes

\section{TIRAGEM}

200

\section{TIPOGRAFIA}

Signika

Bebas Neue

Arial

PAPEL

\section{Capa}

Papel Supremo 250g/m2

Miolo

Papel Offset 90g/m2 